\title{
Atenuação do groundroll utilizando filtragem direcional 2D
}

Paulo, E. M. Melo, Michelângelo, G. Silva, Milton J. Porsani - CPGG-UFBA, Brasil.

\section{Copyright 2006, SBGf - Sociedade Brasileira de Geofísica}

Este texto foi preparado para a apresentação no II Simpósio de Geofísica da Sociedade Brasileira de Geofísica, Natal, 21-23 de setembro de 2006. Seu conteúdo foi revisado pela Comissão Tecno-científica do II SR-SBGf mas não necessariamente representa a opinião da SGBf ou de seus associados. É proibida a reprodução total ou parcial deste material para propósitos comerciais sem prévia autorização da SBGf.

\section{Resumo}

No presente trabalho apresentamos um novo método para atenuação do groundroll. O método utiliza a derivada vertical (temporal) 2D aplicada sobre famílias de traços corrigidos de NMO. Devido a baixa cobertura dos dados de campo utilizado (1200\%) a filtragem foi aplicada sobre famílias de tiro comum, reorganizadas após a aplicação da correção de NMO. Um operador 2D é convolvido com os dados resultando na derivada vertical dos sismogramas. Os coeficientes do operador 2D de filtragem são calculados através de técnica de interpolação baseada no método de empilhamento ótimo proposto por Porsani e Ulrych (1988). Uma aproximação numérica da derivada com relação ao tempo é obtida fazendo a diferença entre os valores interpolados na vizinhança, posterior e anterior, da posição desejada. Resultados numéricos usando o dado sísmico real da bacia sedimentar do Tacutu, mostram que o método proposto é bastante eficaz na atenuação do groundroll, revelando as reflexões mesmo quando estas são mascaradas pelo groundroll. A seção sísmica empiIhada com o método de filtragem direcional (FD) é de qualidade superior aquela obtida com o método convencional de filtragem FK.

\section{Introdução}

Na sísmica de exploração, os ruídos observados nos sismogramas que ocorrem com certa regularidade traço-atraço, são classificados como ruídos coerentes. Dentre esses, merece destaque o groundroll ou ruído de rolamento superficial, presente nos dados sísmicos terrestres, e responsável por uma significativa queda na razão sinal-ruído. O groundroll está associado às ondas superficiais do tipo Rayleigh que ocorrem nas camadas de baixa velocidade próximas à superfície (Yilmaz, 1987). As principais características desse ruído são a alta amplitude, baixa velocidade, dispersão e a concentração de energia nas baixas freqüências.

Os métodos para atenuação do groundrollsão importantes para o processamento de sinais sísmicos. Existem vários métodos disponíveis nos softwares comerciais (PROMAXLandmark, FOCUS-Paradigm). Dentre esses o mais uti- lizado é o método FK (Embree et. al., 1963) que utiliza a transformada 2D de Fourier e que permite filtragem de eventos lineares no tempo, a exemplo do groundroll. Um ponto criticado no método FK é que a filtragem também interfere nas primárias, ou seja, apesar de efetivo na remoção do groundroll o método não consegue revelar as reflexões que foram mascaradas pelo ruído.

O objetivo deste trabalho é apresentar um novo método para atenuação do ruído de rolamento superficial em dados sísmicos terrestres. O método utiliza um operador 2D para obtenção da derivada temporal, aplicado após a correção de NMO. Foi processado o dado sísmico real da bacia sedimentar do Tacutu para comprovar a maior eficácia do método proposto, frente aos resultados obtidos com o método FK.

\section{Metodologia}

A derivada vertical (temporal) em cada ponto de dado é obtida através da convolução do operador de derivada 2D com a matriz que representa o sismograma corrigido de NMO. A correção de NMO tem a finalidade de horizontalizar as reflexões e a operação de derivada vertical tem a função de reforçar as diferenças verticais ao tempo em que atenua os eventos inclinados.

O operador 2D para obtenção da derivada vertical é calculado através da diferença entre os operadores $2 \mathrm{D}$ de interpolação, que predizem imediatamente à frente e atrás do ponto onde se deseja obter o resultado (ponto central do operador).

\section{Método de interpolação}

O problema de obter dados interpolados usando $\mathrm{N}$ amostras conhecidas pode ser matematicamente representada por uma equação linear,

$$
I(\mathbf{r})=\sum_{i=1}^{n} w_{i} A\left(\mathbf{r}_{i}\right)
$$

onde:

$I(\mathbf{r})$ - valor interpolado na posição $\mathbf{r}$,

$A\left(\mathbf{r}_{i}\right)$ - valores conhecidos do dado na posição $\mathbf{r}_{i}$, e

$w_{i}$ - pesos, calculados em função das distâncias

$$
d_{i}=\left|r_{i}-r\right| \text {. }
$$

Uma propriedade desejada em qualquer método de interpolação é a preservação dos dados na sua posição 
original, i.e. $I\left(\mathbf{r}_{i}\right)=A\left(\mathbf{r}_{i}\right)$. Por analogia, com o método proposto por Porsani e Ulrych (1988), para empilhamento ótimo de uma família de traços contaminados com ruído aleatório, os pesos utilizados na interpolação (eq. 1) podem ser obtidos resolvendo-se o sistema de equação:

$$
\left[\begin{array}{cccc}
1+d_{1} & 1 & \cdots & 1 \\
1 & \ddots & \ddots & \vdots \\
\vdots & \ddots & \ddots & 1 \\
1 & \cdots & 1 & 1+d_{N}
\end{array}\right]\left[\begin{array}{c}
w_{1} \\
\vdots \\
\vdots \\
w_{N}
\end{array}\right]=\left[\begin{array}{c}
1 \\
\vdots \\
\vdots \\
1
\end{array}\right]
$$

Notamos que para qualquer $d_{i}=0$, a solução da eq. (2) é dada por: $w_{i}=1$ e $w_{j}=0(j \neq i)$. Também, se $d_{i} \rightarrow \infty$ então $w_{i} \rightarrow 0$. O sistema linear representado pela eq. (2), pode ser resolvida recursivamente com $\left(N^{2} / 2\right)$ operações, utilizando um algoritmo reproduzido abaixo,

$$
\begin{aligned}
& \Delta=1.0 \\
& \text { DO } j=1, N \\
& \qquad \begin{aligned}
w_{j} & =\frac{\Delta}{\Delta+d_{j}} \\
\Delta & =\Delta\left(1.0+w_{j}\right) \\
w_{i} & =w_{i}\left(1.0+w_{j}\right), i=1, \ldots, j-1
\end{aligned}
\end{aligned}
$$

END DO

O algoritmo é numericamente estável desde que utilizemos pontos distintos, o que ocorre no presente caso. Uma vez que $d_{j}(j=1, \ldots, N)$ é positivo, então, todo $w_{j}$ também é positivo.

Nos exemplos apresentados utilizamos um operador 2D $(3 \times 3)$, centrado na posição $(x=0, t=0)$. A Figura 1 mostra o operador $O^{+}$usado para interpolar os dados na vizinhança positiva do tempo, $\left(x=0, t=0^{+}\right)$. Como indicado na Figura, os operadores $O_{1}^{+}, \mathrm{O}_{3}^{+}, \mathrm{O}_{7}^{+}$e $\mathrm{O}_{9}^{+}$ são usados somente nos vértices da matriz de entrada; os operadores $\mathrm{O}_{2}^{+}, \mathrm{O}_{4}^{+}, \mathrm{O}_{6}^{+}$e $\mathrm{O}_{8}^{+}$são usados para interpolar ao longo das laterais da matriz de entrada, e o operador $\mathrm{O}_{5}^{+}$é usado para interpolar todos os pontos restantes, trabalhando no intervalo $\left\{2 \leq x_{i} \leq N_{x}-1\right.$ e $\left.2 \leq t_{i} \leq N_{t}-1\right\}$. Analogamente, um operador $O^{-}=\left\{O_{j}^{-}, j=1, \ldots, 9\right\}$ pode ser formado, para interpolar na vizinhança negativa do tempo, ou seja $\left(x=0, t=0^{-}\right)$.

\section{Obtenção da derivada em relação ao tempo}

Uma aproximação numérica da derivada primeira com relação ao tempo pode ser calculada através da expressão,

$$
\frac{\partial f(x, t)}{\partial t} \approx \frac{I^{+}(x, t)-I^{-}(x, t)}{2 \delta_{t}}
$$

onde $I^{+}(x, t)$ e $I^{-}(x, t)$ representa os valores interpolados nas posições $\mathbf{r}^{+}=\left(x, t+\delta_{t}\right)$ e $\mathbf{r}^{-}=\left(x, t-\delta_{t}\right)$, respectivamente.

A matriz contendo a derivada temporal $\mathbf{X}^{\prime}$ do sismograma pode ser obtida aplicando-se, mediante convolução, separadamente os operadores $\mathrm{O}^{+}$e $\mathrm{O}^{-}$e tomando a diferença do resultado,

$$
\mathbf{X}^{\prime}=\frac{1}{2 \delta t}\left(O^{+} * \mathbf{X}-O^{-} * \mathbf{X}\right)
$$

ou convolvendo a matriz $\mathbf{X}$, diretamente com o operador de derivada primeira,

$$
\mathbf{O}=\frac{\left(O^{+}+O^{-}\right)}{2 \delta t}
$$

O mesmo operador $\mathbf{O}$ pode ser reaplicado sobre $\mathbf{X}^{\prime}$ para obtenção da derivada segunda, ou mesmo reiterado para obtenção de derivadas de mais alta ordem. Ainda, para outras aplicações, o operador de derivada vertical 2D, pode ser aplicado sobre a transposta da matriz $\mathbf{X}$ para gerar a derivada primeira (ou de mais alta ordem) na direção $x$, caso desejado.

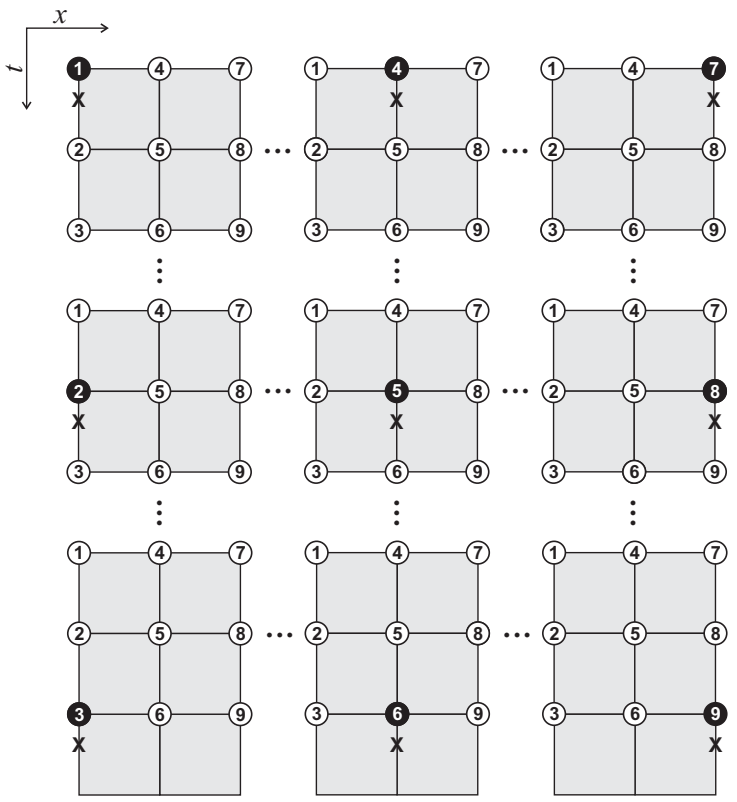

Figura 1: Malha utilizada para o cálculo dos pesos

\section{Exemplos numéricos}

Aplicamos o método FD em dados sísmicos reais da Bacia sedimentar do Tacutu, adquiridos da Ag ência Nacional do Petróleo (ANP). O dado terrestre utilizado foi levantado em 1981 pela PETROBRAS, e contém 179 registros de tiro da linha sísmica RL-5090. A informações de aquisição dessa linha, são: tipo de arranjo split-spread, lanço de 2500150-0-150-2500m, cobertura CMP de $1200 \%$, 96 canais, distância entre geofones de $50 \mathrm{~m}$ e distância entre pontos de tiro igual a $200 \mathrm{~m}$. Os principais problemas desse dado, é o baixo grau de cobertura e a baixíssima razão sinalruído, devido a presença do groundroll.

Antes da aplicação do método FD fizemos um préprocessamento dos dados, envolvendo algumas etapas do processamento sísmico, como a montagem da geometria, a edição, silenciamento dos traços, a análise de velocidade CMP convencional e correção do sobretempo normal. 
A Figura 2 mostra o fluxograma utilizado no processamento.

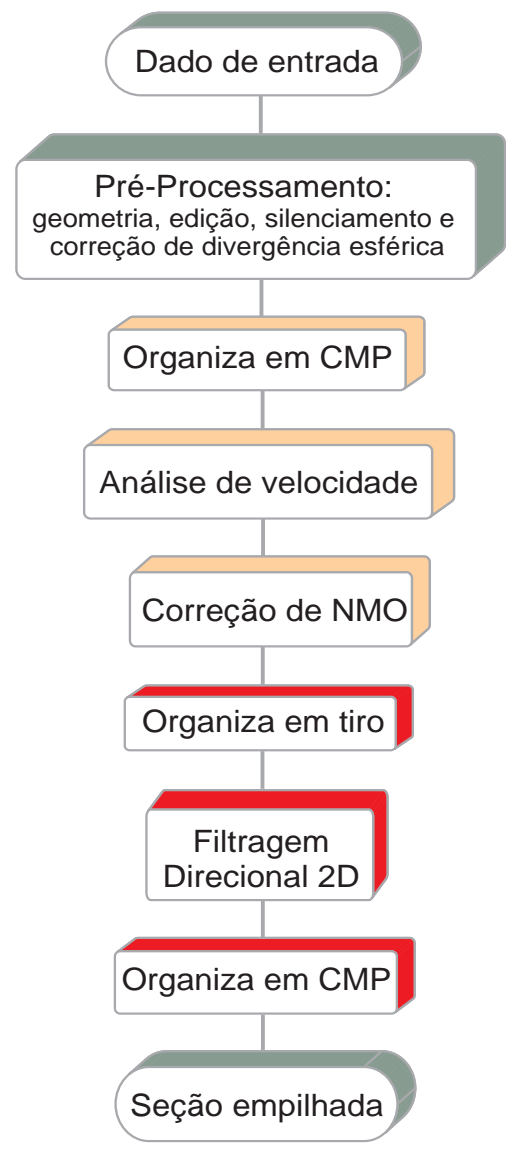

Figura 2: Fluxograma do processamento sísmico mostrando as etapas intermediárias para aplicação do método FD e obtenção da seção empilhada

Como ilustrado no fluxograma, o método FD é aplicado no dado com correção do sobretempo normal usando-se um campo de velocidade. Caso se queira estimar um campo de velocidade mais preciso da subsuperfície, podemos refazer a análise de velocidade utilizando os CMPs filtrados. Com esse procedimento é obtida uma seção com maior resolução, visto que o método melhora qualitativamente a coerência dos traços das família de ponto médio comum.

A Figura 3 mostra o resultado da derivada segunda vertical, reiterando a aplicação do método FD na família de tiro 39 da linha sísmica RL-5090 da Bacia do Tacutu. A filtragem foi aplicada sobre os sismogramas corrigidos do sobretempo normal (Fig. 3a). Observando o sismograma filtrado, (Fig. 3b) vemos que além da remoção do groundroll e da revelação das reflexões, que estavam subjacentes, temos também a remoção do estiramento dos traços, tornando desnecessário o uso do mute strecth no processamento sísmico.

A Figura 4 mostra o resultado obtido com o método FD na família de tiro 39 após a correção de NMO reversa. Observando o sismograma filtrado (Fig. 4c), vemos uma remoção total do groundrolle uma melhor definição e continuidade lateral dos eventos de reflexão, outrora, mascaradas pelo ruído, percebe-se ainda, que a onda direta e refratada também foram atenuadas. Podemos observar no sismograma da filtragem FK, ainda que sutil, a presença do groundroll.

Com o objetivo de verificar o efeito do método FD no conteúdo de freqüência, geramos o espectro de amplitude do dado original, da filtragem com o método $\mathrm{FK}$ e do resultado obtido com o método sob consideração. Comparando as curvas da Figura 5, nota-se que existe uma queda suave na banda de freqüência do groundroll e o aumento da alta freqüência do sinal, para o dado filtrado com o novo método, esse tipo de comportamento não é visto na curva da filtragem FK.

As Figuras 6 e 7 apresentam as seções sísmicas empiIhadas, da linha sísmica RL-5090, após as filtragens FK e FD, respectivamente. O efeito do groundroll, da onda direta e refratada, juntamente com o estiramento dos traços originais, não são totalmente atenuados após a filtragem FK (Figura 6). Na seção empilhada após aplicação da FD (Figura 7), podemos notar uma melhor definição, melhor resolução temporal e melhor continuidade das reflexões. As diferenças entre os dois métodos é mais notada nos eventos localizados próximos à superfície, devido a atenuação do estiramento e maior eficácia na atenuação do groundroll, obtida com o método FD.

\section{Conclusões}

Os resultados obtidos nesse trabalho demonstram que 0 método proposto aplicado nos sismogramas corrigidos do sobretempo normal representa um procedimento de filtragem bastante eficaz para a atenuação do groundroll e do estiramento. Tal procedimento implica na melhoria da resolução temporal e da continuidade lateral dos eventos sísmicos na seção empilhada CMP. A atenuação do groundroll e a boa recuperação das reflexões subjacentes àquele ruído, podem ser aproveitadas para melhorar 0 campo de velocidade, refazendo-se a an álise de velocidades sobre os CMPs filtrados. O operador de filtragem é calculado uma única vez e aplicado em todo o dado, mediante convolução, fazendo com que o custo computacional do novo método seja equivalente ao custo de aplicação do método de Wiener-Levinson para deconvolução do pulso. $\mathrm{O}$ método $\mathrm{FD}$, através da derivada direcional 2D, promove um significativo aumento da resolução temporal e da razão sinal-ruído, como pudemos observar no dado filtrado da bacia do Tacutu. Uma nova estratégia de utilização do método FD pode ser implementada, de forma que seja mapeada outras direções de filtragem. Isto tornaria o método uma poderosa ferramenta para o imageamento direcional de seções e volumes sísmicos, permitindo realçar feições estratigráficas e estruturais de interesse para o geofísico intérprete. 


\section{Agradecimentos}

Os autores agradecem a FINEP, FAPESB, PETROBRAS e CNPq pelo apoio financeiro ao projeto Rede em Risco Exploratório, à Paradigm e Landmark pelas licenças dos softwares de processamento sísmico doadas ao CPGGUFBA.

\section{Referências Bibliográficas}

Embree, P., Burg, J. P. e Backus, M. M. (1963) Wideband velocity filtering - the pie-slice process, Geophysics, v.28(6):948-974.
Halton, L., Worthington, M. H., Makin, J. (1986) Seismic Data Processing, Blackwell Scientific Publications.

Porsani, M. e Ulrych, T. J. (1988) Levinson-type extensions for non-Toeplitz systems with application to optimum stacking, 58th Ann. Internat. Mtg: Soc. of Expl. Geophys., Session:S22.8.

Silva, M. G. (2004) Processamento de dados sísmicos da Bacia do Tacutu, dissertação de mestrado, Universidade Federal da Bahia.

Yilmaz, O. (1987) Seismic Data Processing, Society of Exploration Geophysicists.

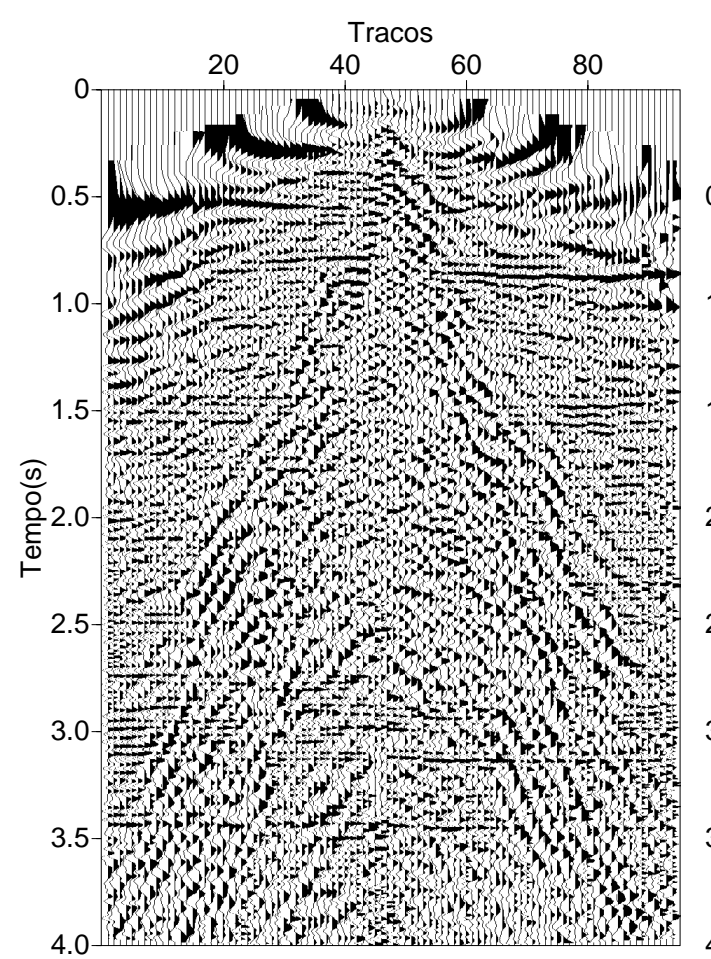

(a)

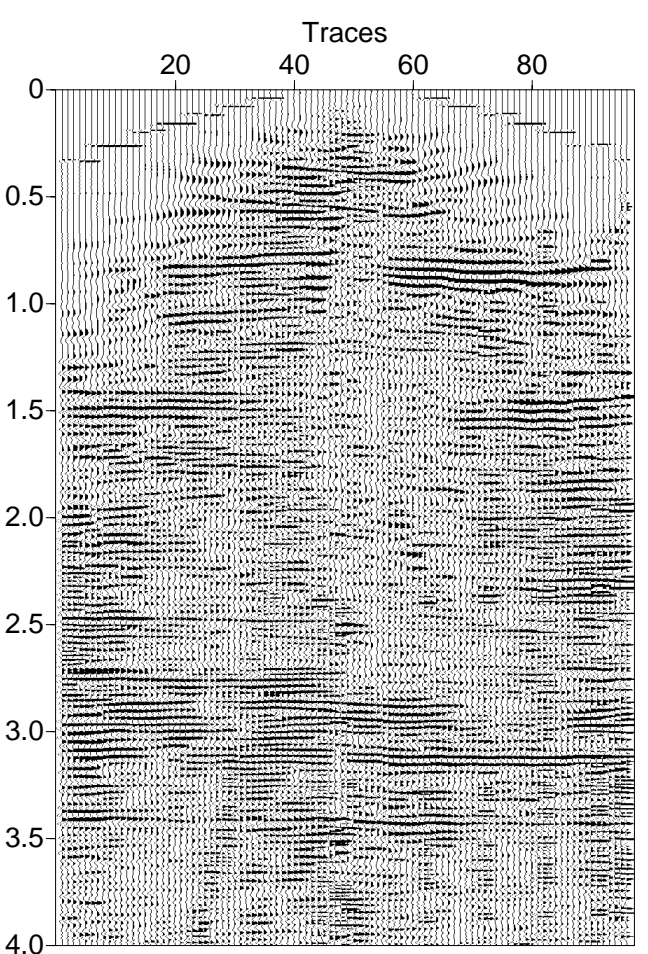

(b)

Figura 3: Sismograma original do tiro 39 após a correção NMO em (a) e o sismograma obtido após a filtragem direcional 2D em (b) 


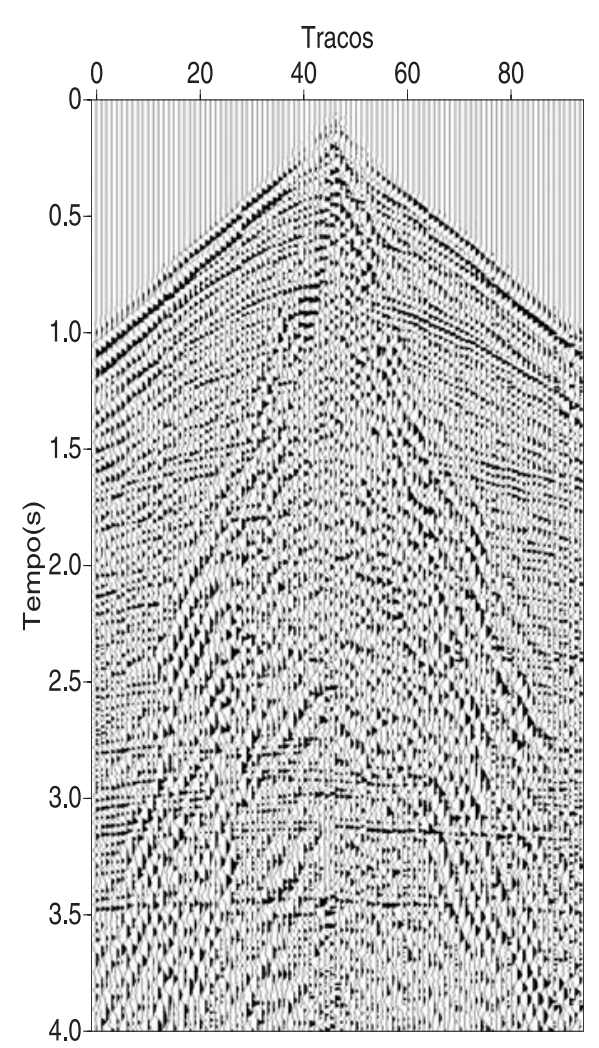

(a)

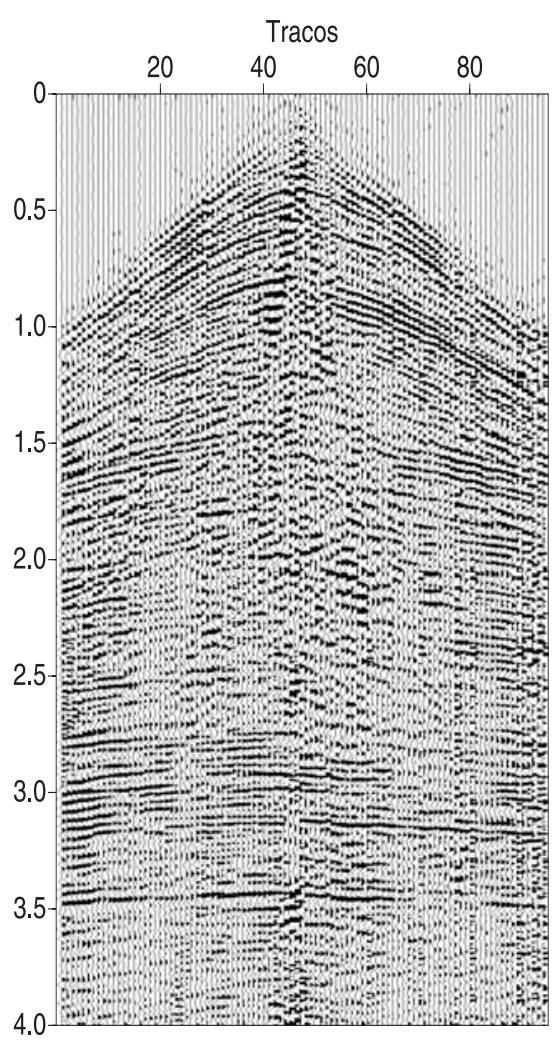

(b)

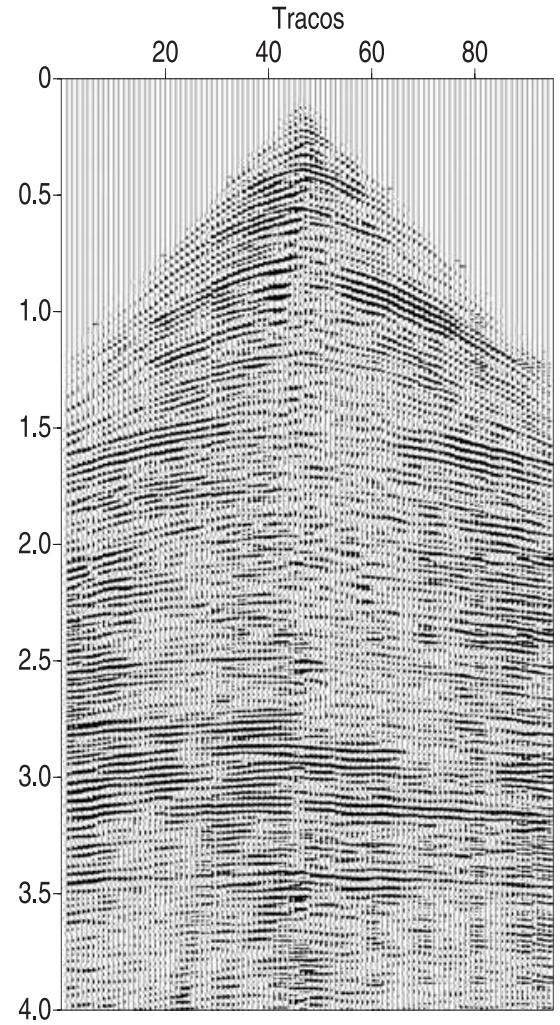

(c)

Figura 4: Resultado da aplicação do método para atenuação do groundroll: sismograma original do tiro 39 em (a), sismograma da filtragem FK em (b) e o sismograma obtido após a filtragem direcional 2D em (c)

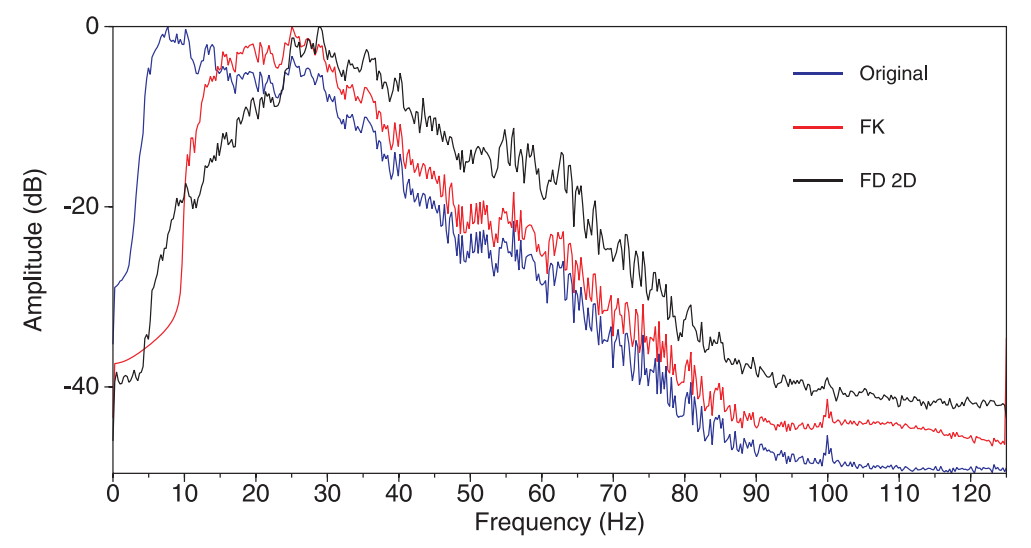

Figura 5: Espectro de amplitude dos sismogramas da figura 4 


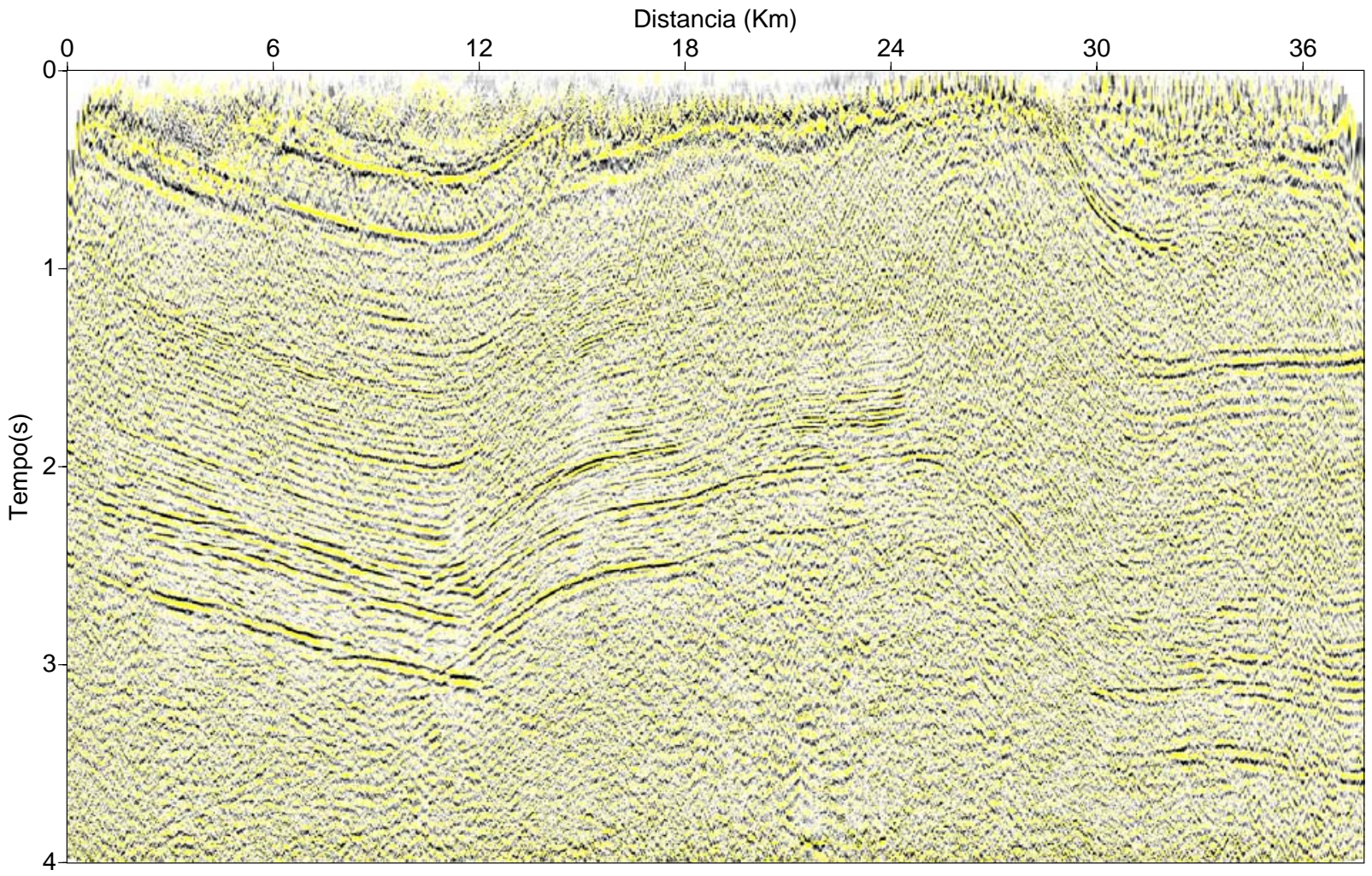

Figura 6: Seção empilhada após a filtragem FK

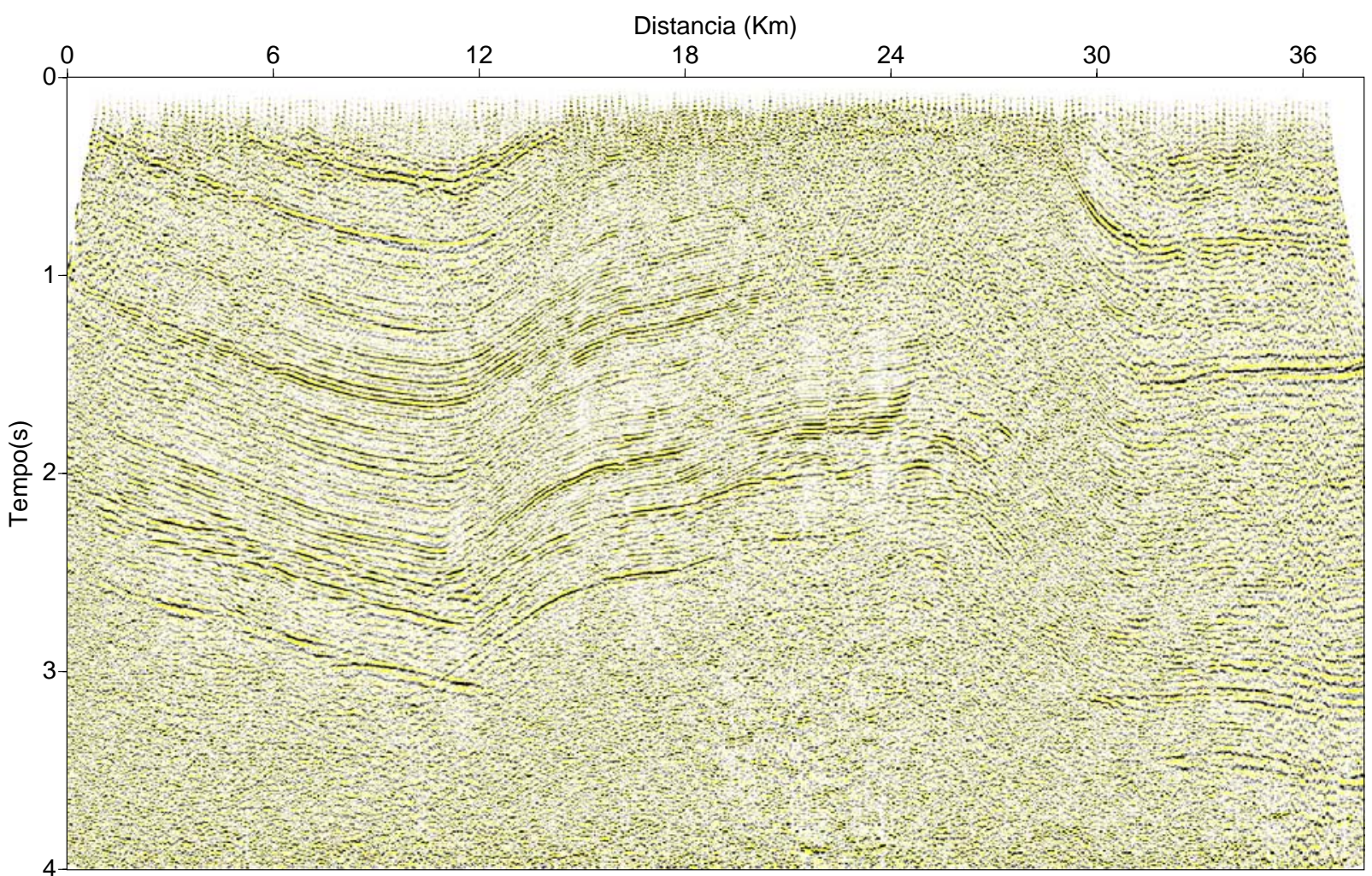

Figura 7: Seção empilhada após a filtragem direcional 2D 